\title{
Quantum Zeno Subspaces induced by Temperature
}

\author{
B. Militello, ${ }^{1}$ M. Scala, ${ }^{1}$ and A. Messina ${ }^{1}$ \\ ${ }^{1}$ Dipartimento di Fisica dell'Università di Palermo, Via Archirafi 36, 90123 Palermo, Itall * $^{\circ}$
}

\begin{abstract}
We discuss the partitioning of the Hilbert space of a quantum system induced by the interaction with another system at thermal equilibrium, showing that the higher the temperature the more effective is the formation of Zeno subspaces. We show that our analysis keeps its validity even in the case of interaction with a bosonic reservoir, provided appropriate limitations of the relevant bandwidth.
\end{abstract}

PACS numbers: $03.65 . \mathrm{Xp}$, 03.65.Aa, 05.30.-d 03.65.-w

\section{INTRODUCTION}

The quantum Zeno effect (QZE), in its original form, is the inhibition of the dynamics of a physical system due to frequent measurements of its state [1]. In fact, the combined action of unitary evolutions for a short time and projection operators gives rise to an effective quantum evolution which becomes closer and closer to a simple projection on the initial state of the system, when the time interval between two measurements becomes shorter and shorter. This effect has been demonstrated in various physical systems [2, 3].

Subsequent studies have raised the problem of analyzing the inhibition of the dynamics induced by continuous measurements, meant as dissipative processes [4]. Indeed, the emission of radiation from a quantum system could be thought of as measurement process, since it says to the observer in which state the system was before the emission. Apart from philosophical discussions, the presence of a strong decay can be responsible for a dynamical decoupling, hindering the dynamics induced by other couplings [5] 8 .

Further developments has brought to the idea that even a unitary coupling can be responsible for the inhibition of the dynamics induced by smaller couplings $[9-11$, eventually leading to the concept of Zeno subspaces, i.e., the formation of suitable invariant subspaces that cancels the effects of weak couplings, as shown by Facchi and Pascazio [12]. The partitioning of the Hilbert space can be obtained also by 'bang-bang' (BB) decoupling. The theories of QZE and BB have been recently unified 1315]. It is worth mentioning that A. Peres has shown how similar behaviors can be observed even in the frame of classical theory [16].

The importance of QZE is related to conceptual aspects and to the foundation of quantum mechanics [17], but it is also witnessed by a variety of applications in the fields of quantum information and nanotechnologies 18 20].

About a decade ago, Ruseckas has studied the influence of the temperature of the detector on the quantum

*Electronic address: bdmilite@fisica.unipa.it
Zeno effect, showing that a higher temperature of the measurement apparatus can enhance the inhibition of the dynamics at short time, then resulting in a more evident Zeno effect by pulsed measurements 21]. More recently, Maniscalco et al have analyzed the crossover from quantum Zeno effect to Inverse Zeno effect (IZE, meant as the enhancement of the dynamics due to repeated measurements [9]) in the Quantum Brownian motion, also bringing to light a certain role of temperature in the occurrence of such effects [22]. Very recently, Bhaktavatsala and Kurizki have analyzed the influence of the statistics of the environment (fermions vs bosons) to the QZE-IZE crossover [23].

In this paper we study the role of temperature in the partitioning of the Hilbert space of a physical system, i.e. we analyze the formation of Zeno subspaces due to the thermal bosons a system is interacting with. Starting from the study - analytical and numerical - of the interaction between a three-state system and a single harmonic oscillator at thermal equilibrium, and between the same three-state system and a finite number of thermalized harmonic oscillators, we find a universal feature corresponding to the partitioning of the Hilbert space of the three-state system at high temperatures. Our analysis allows us to single out a critical temperature for the observation of such a partitioning. The scaling of this threshold temperature easily allows a generalization to the case of infinite harmonic oscillators.

In the next section we show the basic of this effect by studying the single harmonic oscillator case. In the third section, we extend the discussion to the case of a finite but arbitrary number of harmonic oscillators, and in the fourth section we give an estimation of a temperature high enough to observe the formation of Zeno subspaces. Finally, in the last section we provide a detailed discussion of the results.

\section{SINGLE HARMONIC OSCILLATOR}

We consider a three-state system coupled with an harmonic oscillator tuned close to the transition $2 \rightarrow 3$. 
The relevant Hamiltonian is $(\hbar=1)$ :

$$
\begin{aligned}
H & =\sum_{k=1,2,3} \omega_{k}|k\rangle\langle k|+\Omega(|1\rangle\langle 2|+| 2\rangle\langle 1|) \\
& +\omega \hat{a}^{\dagger} \hat{a}+g\left(\hat{a}|2\rangle\left\langle 3\left|+\hat{a}^{\dagger}\right| 3\right\rangle\langle 2|\right),
\end{aligned}
$$

where $|k\rangle$ with $k=1,2,3$ denotes the generic state of the three-state system, $\omega_{k}$ is the relevant energy, $\Omega$ gives the strength of the coupling between 1 and $2, \omega$ is the frequency of the harmonic oscillator (whose annihilation and creation operators are $\hat{a}$ and $\hat{a}^{\dagger}$ ) and $g$ is the coupling strength between the three-state system and the oscillator.

For the sake of simplicity we are assuming that the coupling constant $g$ is real, but all the results we shall show are valid also in the most general case where $g$ is complex and the coupling is $g \hat{a}|2\rangle\left\langle 3\left|+g^{*} \hat{a}^{\dagger}\right| 3\right\rangle\langle 2|$ (see the discussion in the end of Appendix (B).

This Hamiltonian is structured as a set of invariant blocks of the form:

$$
H_{n}=\left(\begin{array}{ccc}
\omega_{1}+n \omega & \Omega & 0 \\
\Omega & \omega_{2}+n \omega & g \sqrt{n+1} \\
0 & g \sqrt{n+1} & \omega_{3}+(n+1) \omega
\end{array}\right),
$$

corresponding to the triplet $|1\rangle|n\rangle,|2\rangle|n\rangle,|3\rangle|n+1\rangle$.

The relevant evolution operator is $U_{n}(t)=$ $\exp \left(-i H_{n} t\right)$. When the condition $g \sqrt{n+1} \gg \Omega$ is fulfilled, such unitary operator does not significantly change the population of the state $|1\rangle|n\rangle$. Qualitatively, we can say that in such case the coupling $\Omega(|1\rangle\langle 2|+| 2\rangle\langle 1|)$ is a small perturbation which does not affect much the dynamics of the state $|1\rangle|n\rangle$ which is an eigenstate in the case $\Omega=0$.

Suppose now that the two subsystems are initially uncorrelated and that the harmonic oscillator is at thermal equilibrium:

$$
\rho=\rho_{A} \otimes \rho_{B},
$$

with

$$
\begin{gathered}
\rho_{A}=|1\rangle\langle 1|, \\
\rho_{B}=\sum_{n} p_{n}|n\rangle\langle n|, \quad p_{n}=Z^{-1} \exp \left(-\frac{n \omega}{k_{B} T}\right),
\end{gathered}
$$

where $Z=\left[1-\exp \left(-\omega /\left(k_{B} T\right)\right)\right]^{-1}$.

The state at time $t$ can be written as:

$$
\rho(t)=\sum_{n} p_{n} U_{n}(t)|n\rangle|1\rangle\langle 1|\langle n| U_{n}^{\dagger}(t),
$$

so that the survival probability of the atomic state $|1\rangle$ is given by:

$$
P(t)=\sum_{n} p_{n}\left|\left\langle 1\left|\left\langle n\left|U_{n}(t)\right| n\right\rangle\right| 1\right\rangle\right|^{2} .
$$

We shall rigorously prove that for any $\varepsilon>0$ it is possible to find a temperature $T_{\varepsilon}$ such that for $T>T_{\varepsilon}$ it is $P(t)>1-\varepsilon$ at every time. In the Appendix B we prove that for any $0<\varepsilon<1$ it is possible to find an index $n_{\varepsilon}$ such that for $n \geq n_{\varepsilon}$ it turns out $\left|\left\langle 1\left|\left\langle n\left|U_{n}(t)\right| n\right\rangle\right| 1\right\rangle\right|^{2} \geq \sqrt{1-\varepsilon}$ for every $t$ (consider the special case $D=1$, with $\left.c_{1}=g \sqrt{n+1}\right)$. Therefore, the survival probability satisfies the following relations:

$$
\begin{aligned}
P(t) & \geq \sum_{n<n_{\varepsilon}} p_{n}\left|\left\langle 1\left|\left\langle n\left|U_{n}(t)\right| n\right\rangle\right| 1\right\rangle\right|^{2} \\
& +\sum_{n \geq n_{\varepsilon}} p_{n} \sqrt{1-\varepsilon} \geq \sum_{n \geq n_{\varepsilon}} p_{n} \sqrt{1-\varepsilon} \\
& =\exp \left(-\frac{\omega n_{\varepsilon}}{k_{B} T}\right) \sqrt{1-\varepsilon},
\end{aligned}
$$

where we have used the relation $\sum_{n \geq n_{\varepsilon}} x^{n}=x^{n_{\varepsilon}} /(1-x)$.

Let us now consider a temperature $T>T_{\varepsilon}=$ $-\left(2 \omega n_{\varepsilon}\right) /\left(k_{B} \log (1-\varepsilon)\right)$, for which one has $\exp \left(-\omega n_{\varepsilon} /\left(k_{B} T_{\varepsilon}\right)\right)>\sqrt{1-\varepsilon}$. Then, for such high temperatures one has $P(t)>1-\varepsilon$ for any $t$. This is a clear manifestation of the formation of temperatureinduced Zeno subspaces. Indeed, the higher the temperature, the more the coupling between atomic states $|1\rangle$ and $|2\rangle$ is neutralized by the coupling between $|2\rangle$ and $|3\rangle$ mediated by the harmonic oscillator.

It is important to note that when $T \approx 0$ the only block really involved in the dynamics is the one corresponding to $n=0$, and the inhibition of the time evolution can occur only if $g \gg \Omega$. On the contrary, when the temperature increases, there are a number of blocks effectively involved in the dynamics, and in most of them it happens that the coupling constant $g \sqrt{n+1}$ exceeds $\Omega$, even if $g$ itself is not large. In the $T \rightarrow \infty$ limit all the blocks (an infinite number) are involved and in the majority of them the condition $g \sqrt{n+1} \gg \Omega$ is fulfilled.

To support our previous analysis, we show in Fig. 1 the evolution of the survival probability of the state $|1\rangle$ when the system is interacting with an oscillator resonant to the transition $2 \rightarrow 3$, for different temperatures. It is well visible that as the temperature increases the survival probability tends toward unity at every time.

\section{MANY HARMONIC OSCILLATORS}

The previous result can be generalized to a set of $D$ harmonic oscillators interacting with a three-state system. The relevant Hamiltonian reads:

$$
\begin{aligned}
H & =\sum_{k=1,2,3} \omega_{k}|k\rangle\langle k|+\Omega(|1\rangle\langle 2|+| 2\rangle\langle 1|) \\
& +\sum_{k=1}^{D} \tilde{\omega}_{k} \hat{a}_{k}^{\dagger} \hat{a}_{k}+\sum_{k=1}^{D} g_{k}\left(\hat{a}_{k}|2\rangle\left\langle 3\left|+\hat{a}_{k}^{\dagger}\right| 3\right\rangle\langle 2|\right) .
\end{aligned}
$$




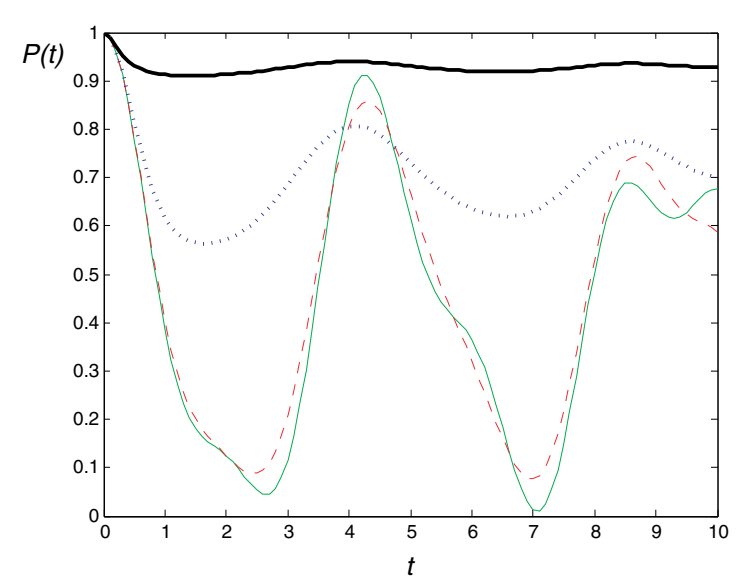

FIG. 1: (Color online) The survival probability of the atomic state $|1\rangle$ as a function of time (in units of $\Omega^{-1}$ ), at different temperatures: $k_{B} T / \omega_{23}=0.1$ (green solid line), $k_{B} T / \omega_{23}=1$ (red dashed line), $k_{B} T / \omega_{23}=10$ (blue dotted line), $k_{B} T / \omega_{23}=100$ (black bold line). Here $\omega_{1} / \Omega=20$, $\omega_{2} / \Omega=19, \omega_{3}=0, \omega_{23}=\omega_{2}-\omega_{3}, D=1, g / \Omega=1$. The harmonic oscillator is tuned to the $2 \rightleftarrows 3$ transition.

$$
H_{n_{1} \ldots n_{D}}=\left(\begin{array}{cc}
\omega_{1}+\delta & \Omega \\
\Omega & \omega_{2}+\delta \\
0 & g_{1} \sqrt{n_{1}+1} \\
\vdots & \vdots \\
0 & g_{D} \sqrt{n_{D}+1}
\end{array}\right.
$$

with $\delta=\sum_{k} n_{k} \tilde{\omega}_{k}$. The only non vanishing terms of this matrix belong to the second row, to the second column (all the couplings involve state $|2\rangle\left|n_{1}, \ldots n_{D}\right\rangle$ ) and to the diagonal (free energies). Similarly to the case of one harmonic oscillator, when it happens that $g_{k} \sqrt{n_{k}+1} \gg \Omega \forall k$, one can assume that the Hamiltonian in (10) with $\Omega=0$ is the 'unperturbed' Hamiltonian, which has $|1\rangle\left|n_{1}, \ldots n_{D}\right\rangle$ as eigenstate; then one can consider the effects of the perturbation (the coupling $\Omega(|1\rangle\langle 2|+| 2\rangle\langle 1|))$, which does not change much the previous situation, leaving the state $|1\rangle\left|n_{1}, \ldots, n_{D}\right\rangle$ as an eigenstate up to terms of the order $\Omega /\left(g_{k} \sqrt{n_{k}+1}\right)$. This implies that for very large $n_{1}, n_{2}, \ldots n_{D}$ the population of the state $|1\rangle\left|n_{1}, \ldots n_{D}\right\rangle$ does not significantly change.

The circumstance that the state $|1\rangle\left|n_{1}, \ldots, n_{D}\right\rangle$ is close to an eigenstate of the Hamiltonian for large excitation numbers is rigorously proven in the Appendix B.

Similarly to the case of a single harmonic oscillator, for large enough temperature the blocks with large excitation numbers are more and more involved in the dynamics and the subspace separation becomes more and more significant.

In Fig. 2 we show the time evolution of the survival
Each set $\quad\left\{|1\rangle\left|n_{1}, n_{2}, \ldots, n_{D}\right\rangle, \quad|2\rangle\left|n_{1}, n_{2}, \ldots, n_{D}\right\rangle\right.$, $\left.|3\rangle\left|n_{1}+1, n_{2}, \ldots, n_{D}\right\rangle, \quad \ldots|3\rangle\left|n_{1}, n_{2}, \ldots, n_{D}+1\right\rangle\right\}$ individualizes an invariant subspace, corresponding to the following invariant block: probability of the state $|1\rangle$ when the three-state system is interacting with a finite number of oscillators having frequencies close to the $2 \rightarrow 3$ transition. In these calculations we have taken $g=0.5$ and $D=4$, in order to keep constant the quantity $g^{2} D$, which resembles the zerotemperature decay rate in a bath $\Gamma=g^{2}(\omega) D(\omega)$, where $D(\omega)$ is the density of modes of frequency $\omega[24,25]$. In order to better afford numerical calculations in the presence of a larger Hilbert space, we have considered values of the temperature lower than those considered in the case $D=1$. The Zeno-like effect is still appreciable anyway. Indeed, similarly to the case of a single harmonic oscillator, the trend of the survival probability as temperature increases is well visible.

\section{THRESHOLD TEMPERATURE}

Let us now study in detail the dependence of the appearance of Zeno subspaces on the temperature.

Generally speaking, if the initial state of a quantum system has an overlap $\sqrt{\chi} \mathrm{e}^{i \varphi}$ with an eigenstate of the Hamiltonian, such that $\chi>1 / 2$, then the survival proba- 


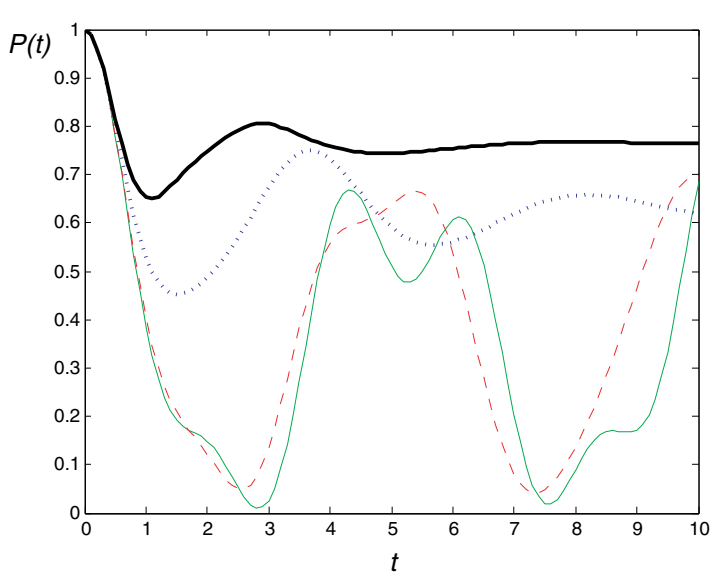

FIG. 2: (Color online) The survival probability of the atomic state $|1\rangle$ as a function of time (in units of $\Omega^{-1}$ ), at different temperatures: $k_{B} T / \omega_{23}=0.1$ (green solid line), $k_{B} T / \omega_{23}=1$ (red dashed line), $k_{B} T / \omega_{23}=5$ (blue dotted line), $k_{B} T / \omega_{23}=$ 10 (black bold line). Here $\omega_{1} / \Omega=20, \omega_{2} / \Omega=19, \omega_{3}=0$, $\omega_{23}=\omega_{2}-\omega_{3}, D=4, g_{k} / \Omega=g / \Omega=0.5$. The four oscillators are tuned to the frequencies: $\tilde{\omega}_{1}=\omega_{23}, \tilde{\omega}_{2} \approx 0.996 \omega_{23}, \tilde{\omega}_{3} \approx$ $0.992 \omega_{23}, \tilde{\omega}_{4} \approx 0.987 \omega_{23}$.

bility can never be smaller than $(\chi-(1-\chi))^{2}=(2 \chi-1)^{2}$. We prove this statement in the Appendix $\mathrm{A}$

From Appendix $\mathrm{B}$ we know that in a given block characterized by $n_{1}, n_{2}, \ldots, n_{D}$ with $c \equiv \sum_{l} g_{l}^{2} n_{l}$ large enough, there exists an eigenstate, say $\left|\Psi_{n 1, \ldots, n_{D}}\right\rangle$, whose overlap with the state $\left|A_{n_{1}, n_{2}, \ldots, n_{D}}\right\rangle \equiv|1\rangle\left|n_{1}, n_{2}, \ldots, n_{D}\right\rangle$ is:

$$
\begin{aligned}
& \left|\left\langle\Psi_{n 1, \ldots, n D} \mid A_{n_{1}, n_{2}, \ldots, n_{D}}\right\rangle\right| \\
& \geq \frac{m c^{2}}{\left(m^{2} c^{4}+16 M^{2} \Omega^{2} c^{2}+4 m^{2} M^{2} \Omega^{2}\right)^{1 / 2}},
\end{aligned}
$$

where $m=\min _{j}\left(\left|\omega_{3}-\omega_{1}+\tilde{\omega}_{j}\right|\right)$ and $M=\max _{j}\left(\mid \omega_{3}-\right.$ $\left.\omega_{1}+\tilde{\omega}_{j} \mid\right)$.

To obtain the previous formula, according to our analysis in Appendix B. we require either $\tilde{\omega}_{j}>\omega_{1}-\omega_{3} \quad \forall k$ or $\tilde{\omega}_{j}<\omega_{1}-\omega_{3} \quad \forall k$, and $m>0$ anyway. For

$$
c \geq c_{\varepsilon}=\frac{4 M \Omega}{m} \sqrt{\frac{1+(1-\varepsilon)^{1 / 4}}{1-(1-\varepsilon)^{1 / 4}}} \approx \frac{8 \sqrt{2} \Omega M}{m} \varepsilon^{-\frac{1}{2}},
$$

the squared modulus of the overlap is larger than $\chi=$ $\left((1-\varepsilon)^{1 / 4}+1\right) / 2$, so that the survival probability of the state $\left|A_{n_{1}, n_{2}, \ldots, n_{D}}\right\rangle$ can never be smaller than $\sqrt{1-\varepsilon}$.

Let us consider the part of the bosonic Hilbert space made of those invariant subspaces where it happens that the relevant $c$ is smaller than $c_{\varepsilon}$, and introduce the relevant partition function:

$$
\begin{aligned}
Z_{\varepsilon} & \equiv Z\left(\sum_{j} g_{j}^{2} n_{j}<c_{\varepsilon}^{2}\right) \\
& =\sum_{\sum_{k} g_{k}^{2} n_{k}<c_{\varepsilon}^{2}} \mathrm{e}^{-\sum_{k} \tilde{\omega}_{k} n_{k} /\left(k_{B} T\right)} .
\end{aligned}
$$

The smaller $Z_{\varepsilon}$ the more the atomic state $|1\rangle$ does not evolve, and it turns out that such a state is essentially separated from the other two atomic states.

In the following we will show that the contribution of $Z_{\varepsilon}$ to the total partition function becomes negligible in the limit $T \rightarrow \infty$.

Calculation on the hypercube - The condition $c \geq c_{\varepsilon}$ is surely guaranteed if there is at least one boson number $n_{k} \geq n_{\varepsilon}$, where $n_{\varepsilon}$ is the smallest integer number larger than $c_{\varepsilon}^{2} / g_{\min }^{2}$ with $g_{\min }=\min _{j}\left|g_{j}\right|$. Therefore, the condition $c \geq c_{\varepsilon}$ is a fortiori satisfied if $n_{k} \geq n_{\varepsilon} \quad \forall k$.

The ratio between $Z_{\varepsilon}$ and the total partition function is:

$$
\frac{Z_{\varepsilon}}{Z_{\mathrm{tot}}} \leq \frac{Z\left(\left\{n_{j}<n_{\varepsilon}\right\}\right)}{Z_{\mathrm{tot}}}=\prod_{j=1}^{D}\left(1-x_{j}^{n_{\varepsilon}}\right),
$$

where $Z\left(\left\{n_{j}<n_{\varepsilon}\right\}\right)$ is meant as the partition function corresponding to $n_{j}<n_{\varepsilon} \forall j$ :

$$
\begin{aligned}
Z\left(\left\{n_{j}<n_{\varepsilon}\right\}\right) & =\prod_{j=1}^{D}\left(\sum_{n_{j}<n_{\varepsilon}} \mathrm{e}^{-\tilde{\omega}_{j} n_{j} /\left(k_{B} T\right)}\right) \\
& =\prod_{j=1}^{D} \frac{1-x_{j}^{n_{\varepsilon}}}{1-x_{j}}
\end{aligned}
$$

while

$$
Z_{\mathrm{tot}}=\prod_{j=1}^{D}\left(1-x_{j}\right)
$$

with $x_{j}=\exp \left(-\tilde{\omega}_{j} /\left(k_{B} T\right)\right)$. Introducing $\tilde{\omega}_{\max }=$ $\max _{j} \tilde{\omega}_{j}$ and $x \equiv \min _{j} x_{j}=\exp \left(-\tilde{\omega}_{\max } /\left(k_{B} T\right)\right)$, one finds

$$
\frac{Z_{\varepsilon}}{Z_{\mathrm{tot}}} \leq\left(1-x^{n_{\varepsilon}}\right)^{D}
$$

The request $Z_{\varepsilon} / Z_{\text {tot }}<1-\sqrt{1-\varepsilon} \equiv \alpha_{\varepsilon}$, translates into

$$
T>-\frac{\tilde{\omega}_{\max } n_{\varepsilon}}{k_{B} \log \left(1-\alpha_{\varepsilon}^{1 / D}\right)} .
$$

In the limit of small $\varepsilon$ we have that $c_{\varepsilon}$ scales as $8 \sqrt{2} \Omega M m^{-1} \varepsilon^{-1 / 2}$ and that $\log \left(1-\alpha_{\varepsilon}^{1 / D}\right) \approx-(\varepsilon / 2)^{1 / D}$ provided $D$ is not too large (we need $\alpha_{\varepsilon}^{1 / D} \ll 1$ ). Therefore, under such hypotheses, it is sufficient to have:

$$
T>T_{\mathrm{c}}(\varepsilon) \approx \frac{2^{6} M^{2} \Omega^{2} \tilde{\omega}_{\max }}{m^{2} g_{\min }^{2} k_{B}}\left(\frac{2}{\varepsilon}\right)^{\frac{D+1}{D}} D,
$$

which eventually guarantees that the survival probability of the state $|1\rangle$ is always larger than $1-\varepsilon$.

Calculation on the hypersphere - Let us now calculate the $Z_{\varepsilon}$ partition function using the approximation to the continuum, which better applies for high temperature (so 
that the Boltzmann factors do not change much with respect to the $n_{j}$ 's):

$$
\begin{aligned}
Z_{\varepsilon} & =\sum_{\sum_{k} g_{k}^{2} n_{k}<c_{\varepsilon}^{2}} \mathrm{e}^{-\sum_{j} \tilde{\omega}_{j} n_{j} /\left(k_{B} T\right)} \\
& \approx \int \ldots \int_{\sum_{n} y_{n}^{2}<c_{\varepsilon}^{2}} \mathrm{e}^{-\sum_{j} \tilde{\omega}_{j} n_{j} /\left(k_{B} T\right)} \prod_{n=1}^{D} \frac{2 y_{n} \mathrm{~d} y_{n}}{g_{n}^{2}},
\end{aligned}
$$

where we have introduced the variables $y_{k}=g_{k} \sqrt{n_{k}}$, so that $\mathrm{d} n_{k}=2 g_{k}^{-2} y_{k} \mathrm{~d} y_{k}$.

In the large temperature limit the exponential in Eq. (20) approaches unity and it turns out to be:

$$
\frac{Z_{\varepsilon}}{Z_{\mathrm{tot}}} \approx \frac{2^{D} G_{D} c_{\varepsilon}^{2 D}}{\prod_{n} g_{n}^{2}} \frac{\prod_{n} \tilde{\omega}_{n}}{\left(k_{B} T\right)^{D}},
$$

where $G_{D}=\left(2^{D} D !\right)^{-1}$ is the appropriate geometric factor (see the appendix $(\mathrm{C}$ ), and where we have taken the high temperature limit for the total partition function: $Z_{\mathrm{tot}} \approx \prod_{j}\left[\tilde{\omega}_{j} /\left(k_{B} T\right)\right]$.

The condition $Z_{\varepsilon} / Z_{\mathrm{tot}}<\alpha_{\varepsilon}$ then becomes:

$$
T>2 G_{D}^{1 / D} \frac{c_{\varepsilon}^{2} \tilde{\omega}_{\mathrm{av}}}{k_{B} g_{\mathrm{av}}^{2}} \alpha_{\varepsilon}^{-1 / D},
$$

where $g_{\mathrm{av}}=\left(\prod_{n} g_{n}\right)^{1 / D}$ and $\tilde{\omega}_{\mathrm{av}}=\left(\prod_{n} \tilde{\omega}_{n}\right)^{1 / D}$.

By considering the expression of $c_{\varepsilon}$ for small $\varepsilon$ and that of $G_{D}$ after the Stirling approximation, and taking into account that for large $D$ one has $(\sqrt{2 \pi D})^{1 / D} \rightarrow 1$ and $\alpha_{\varepsilon}^{-1 / D} \rightarrow 1$, we can just ask:

$$
T>T_{\mathrm{s}}(\varepsilon) \equiv \frac{2^{7} \text { e } M^{2} \Omega^{2} \tilde{\omega}_{\mathrm{av}}}{m^{2} g_{\mathrm{av}}^{2} k_{B} D} \varepsilon^{-1},
$$

which guarantees that the survival probability of the state $|1\rangle$ is always larger than $1-\varepsilon$. In order to ensure the validity of the previous calculations, condition (23) must always be associated to the high temperature limit condition, $k_{B} T \gg \tilde{\omega}_{\max }$, which allows the passage to continuum in Eq. (20)

Apart from the technical aspects of the development of the calculations (on the hypercube and on the hypersphere), the two threshold temperatures differ because of the regime where they apply and because of the scaling with the number of oscillators. In particular, $T_{\mathrm{c}}(\varepsilon)$ applies when the number of oscillators is small, and turns out to be proportional to such number. We do not need such a threshold temperature to be much higher than the frequencies of the oscillators. On the contrary, $T_{\mathrm{S}}(\varepsilon)$ applies in the limit of high temperature and can be applied even in the case of large number of oscillators. Such threshold temperature scales as the inverse of the number of oscillators. This means that by assuming that the coupling constants scale as $g_{k} \sim D^{-1 / 2}$, one obtains that the threshold temperature $T_{\mathrm{s}}(\varepsilon)$ is more or less independent of the number of oscillators.

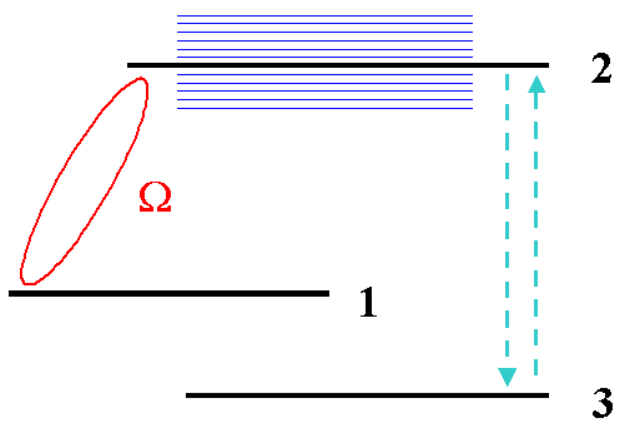

(a)

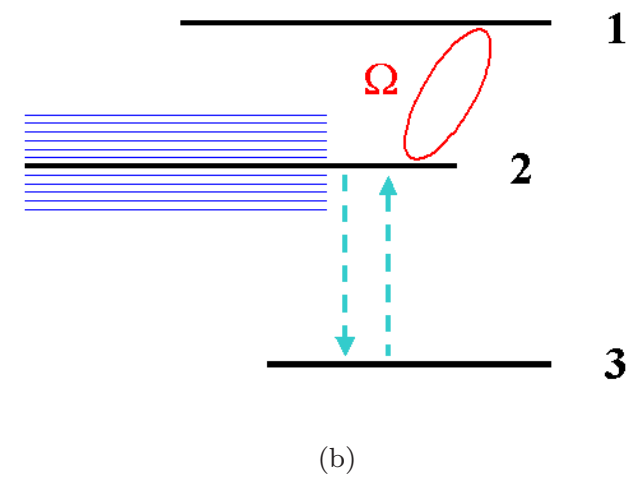

FIG. 3: (Color online). The two possible physical scenarios corresponding to a bandwidth which does not intersect the Bohr frequency associated to the $1 \rightleftarrows 3$ transitions. In (a) all the frequencies of the oscillators exceed the frequency $\omega_{1}-\omega_{3}$, while in (b) they are always smaller. The numerical simulations in Fig. 1 and Fig. 2 are both obtained in the scenario (b).

It is worth stressing that, according to the analysis reported in the appendix $\mathrm{B}$ the previous treatment is valid if one of these two conditions is satisfied: either $\tilde{\omega}_{k}>\omega_{1}-\omega_{3} \quad \forall k$ or $\tilde{\omega}_{k}<\omega_{1}-\omega_{3} \quad \forall k$, always requiring $m>0$. This means that we have to avoid those situations wherein some frequencies of the harmonic oscillators are smaller than $\omega_{1}-\omega_{3}$ while some other are larger. In other words, the Bohr frequency of the transition $1 \rightarrow 3$ should not belong to the frequency band of the oscillators. The two possible scenarios are pictured in Fig. 3

Finally, we emphasize that usually $T_{\mathrm{c}}(\varepsilon)$ significantly overestimates the temperature necessary to have the formation of Zeno subspaces. Moreover, both $T>T_{\mathrm{c}}(\varepsilon)$ and $T>T_{\mathrm{S}}(\varepsilon)$ provide only sufficient conditions, so that in principle the Zeno subspaces can appear for lower temperatures.

\section{DISCUSSION}

In this paper we have analyzed a simple interaction model between a set of harmonic oscillators initially at 
thermal equilibrium and a three-state system. The latter system is prepared in a state (1) which is not directly involved in boson-assisted transitions, but it is coupled to a state (2) which undergoes transitions toward the third state (3) due to the interaction with the bosonic part of the system. Therefore there is a coupling between states 2 and 3 mediated by the harmonic oscillators responsible for transitions between such two states. The states 1 and 2 are directly coupled, but when the coupling between 2 and 3 mediated by the oscillators becomes very strong, a partitioning of the Hilbert space neutralizes the direct coupling between 1 and 2 .

There are two ways to make the coupling between 2 and 3 stronger: one possibility is to consider higher coupling constants $g_{k}$ 's, which makes the effect occur even at zero temperature; another possibility is to increase the temperature of the harmonic oscillators in order to make that those bosonic states which stronger couple the transitions $2 \rightleftarrows 3$ (due to an high value of $g_{k} \sqrt{n_{k}+1}$ ) are much involved in the dynamics. The latter case is the one considered in this paper.

A very remarkable result is the scaling of the threshold temperature at which the Zeno subspaces surely appear. Indeed, in the limit of high temperature such threshold temperature scales as $g_{\mathrm{av}}^{-2} D^{-1}$. Therefore, assuming a larger and larger number of oscillators, keeping constant the quantity $g_{\mathrm{av}}^{2} D$, we can rigorously assert that even in the presence of a bosonic bath the partitioning of the Hilbert space occurs. Instead, if the coupling constants $g_{k}$ 's scale in such a way that $g_{\mathrm{av}}^{2} D$ significantly decreases when $D$ increases, then the temperature necessary to create the Zeno subspaces becomes higher and higher and could become infinite when the number of modes of the bosonic field is infinite.

Concerning the limitations of our analysis, it is important to clarify that the proof given in the appendix $B$ is based on a precise hypothesis about the diagonal entries of the matrix. Such hypothesis translates into the assumption that the frequencies of the oscillators are localized in a finite band, so that it is possible to find a finite maximum and a non-vanishing minimum - both of the same sign - for the Bohr frequencies related to transitions from state 1 to state 3 assisted by the acquisition of a bosonic quantum. In other words, the Bohr frequency $\omega_{1}-\omega_{3}$ should not be included in the frequency band that contains all the frequencies of the harmonic oscillators. Therefore, the $D \rightarrow \infty$ limit should be considered keeping finite the bandwidth of the reservoir and ensuring that such band does not intersect the Bohr frequency $\omega_{1}-\omega_{3}$.

As a final remark, we mention that if the subsystem $B$ is made of spins instead of harmonic oscillators, the previous effect seemingly does not occur. Indeed, for the partitioning to happen we need to have $g_{k} \sqrt{n_{k}+1} \gg \Omega$, which is fulfilled for large $n_{k}$, and then in the majority of the invariant subspaces. Since such large numbers of excitations are not possible for a single spin, we guess that it is more difficult to obtain Zeno subspaces through the interaction with spins at thermal equilibrium, at least assuming a structure of interaction analogous to the one we considered in this paper.

\section{Appendix A}

Let $|\psi(0)\rangle$ be the initial state of the physical system, $\left|\phi_{k}\right\rangle$ the eigenstates of the Hamiltonian. Introducing $\left\langle\psi(0) \mid \phi_{k}\right\rangle=\sqrt{\chi_{k}} \mathrm{e}^{i \alpha_{k}}$, one can write:

$$
|\psi(0)\rangle=\sum_{k} \sqrt{\chi_{k}} \mathrm{e}^{-i \alpha_{k}(0)}\left|\phi_{k}\right\rangle
$$

which evolves into the state

$$
|\psi(0)\rangle=\sum_{k} \sqrt{\chi_{k}} \mathrm{e}^{-i \alpha_{k}(t)}\left|\phi_{k}\right\rangle
$$

If there is a $\chi_{\bar{k}}$, say $\chi_{1}$, which is larger than the sum of all the remaining ones, then it is easy to convince oneself of the following relations:

$$
\begin{aligned}
|\langle\psi(0) \mid \psi(t)\rangle| & =\left|\chi_{1}+\sum_{k \neq 1} \chi_{k} \mathrm{e}^{-i\left[\alpha_{k}(t)-\alpha_{1}(t)\right]}\right| \\
& \geq\left|\chi_{1}-\sum_{k \neq 1} \chi_{k}\right|=\left|2 \chi_{1}-1\right|,
\end{aligned}
$$

where we have used $\sum_{k} \chi_{k}=1$ and we have considered the 'worst case' corresponding to $\alpha_{k}(t)-\alpha_{1}(t)=\pi$ $\forall k \neq 1$.

The condition $\chi_{1}>\sum_{k \neq 1} \chi_{k}$ is clearly equivalent to $\chi_{1}>1 / 2$. The survival probability is the squared modulus of the overlap of the initial state and the evolved state:

$$
|\langle\psi(0) \mid \psi(t)\rangle|^{2} \geq\left(2 \chi_{1}-1\right)^{2}
$$

\section{Appendix B}

Let us consider an Hamiltonian of the following form:

$$
H=\left(\begin{array}{ccccc}
\delta_{1} & \Omega & 0 & \ldots & 0 \\
\Omega & \delta_{2} & c_{1} & \ldots & c_{D} \\
0 & c_{1} & \delta_{3} & \ldots & 0 \\
\vdots & \vdots & \vdots & \ddots & \vdots \\
0 & c_{D} & 0 & \ldots & \delta_{D+2}
\end{array}\right)
$$

in the basis $|A\rangle,|B\rangle,\left|C_{1}\right\rangle, \ldots\left|C_{D}\right\rangle$. Let us assume $\delta_{k} \neq$ $0 \forall k$ and introduce the quantities $c=\left(\sum_{k=1}^{D} c_{k}^{2}\right)^{1 / 2}, m \equiv$ $\min _{k=1}^{D}\left(\left|\delta_{k+2}-\delta_{1}\right|\right)$ and $M \equiv \max _{k=1}^{D}\left(\left|\delta_{k+2}-\delta_{1}\right|\right)$. For the sake of simplicity, we are considering the $c_{k}$ 's as real quantities.

In this appendix we prove that in the limit of very large $c$ there is an eigenstate which approaches the state $|A\rangle$, provided $m>0$. In particular, we will se that there exists an eigenvalue $\lambda_{1}$ such that $\left|\lambda_{1}-\delta_{1}\right|<2 M \Omega^{2} / c^{2}$, and that the corresponding eigenstate $\left|\lambda_{1}\right\rangle$ has an overlap with $|A\rangle$ which becomes closer and closer to unity as $c$ increases. 
The secular equation - The determinant of the matrix above can be developed (with respect to the first row) as follows:

$$
\operatorname{det} H=\delta_{1}\left(\begin{array}{cccc}
\delta_{2} & c_{1} & \ldots & c_{D} \\
c_{1} & \delta_{3} & \ldots & 0 \\
\vdots & \vdots & \ddots & \vdots \\
c_{D} & 0 & \ldots & \delta_{D+2}
\end{array}\right)-\Omega\left(\begin{array}{cccc}
\Omega & c_{1} & \ldots & c_{D} \\
0 & \delta_{3} & \ldots & 0 \\
\vdots & \vdots & \ddots & \vdots \\
0 & 0 & \ldots & \delta_{D+2}
\end{array}\right)
$$

where the second minor can be easily evaluated as $\Omega \prod_{k=3}^{D+2} \delta_{k}$, while the first minor is calculated as follows:

$$
\begin{gathered}
\delta_{2}\left(\begin{array}{ccccc}
\delta_{3} & 0 & 0 & \ldots & 0 \\
0 & \delta_{4} & 0 & \ldots & 0 \\
0 & 0 & \delta_{5} & \ldots & 0 \\
\vdots & \vdots & \vdots & \ddots & \vdots \\
0 & 0 & 0 & \ldots & \delta_{D+2}
\end{array}\right)-c_{1}\left(\begin{array}{ccccc}
c_{1} & 0 & 0 & \ldots & 0 \\
c_{2} & \delta_{4} & 0 & \ldots & 0 \\
c_{3} & 0 & \delta_{5} & \ldots & 0 \\
\vdots & \vdots & \vdots & \ddots & \vdots \\
c_{D} & 0 & 0 & \ldots & \delta_{D+2}
\end{array}\right)+c_{2}\left(\begin{array}{ccccc}
c_{1} & \delta_{3} & 0 & \ldots & 0 \\
c_{2} & 0 & 0 & \ldots & 0 \\
c_{3} & 0 & \delta_{5} & \ldots & 0 \\
\vdots & \vdots & \ddots & \vdots \\
c_{D} & 0 & \ldots & \delta_{D+2}
\end{array}\right)+\ldots \\
= \\
\prod_{k=2}^{D+2} \delta_{k}-c_{1}^{2} \prod_{k=4}^{D+2} \delta_{k}-c_{2}^{2} \times \prod_{k=3,5,6 \ldots D+2} \delta_{k}+\ldots=\left(\prod_{k=2}^{D+2} \delta_{k}\right) \times\left(1-\sum_{l=1}^{D} \frac{c_{l}^{2}}{\delta_{2} \delta_{l+2}}\right) .
\end{gathered}
$$

Taking into account these results, one obtains:

$$
\operatorname{det} H=\left(\prod_{k=1}^{D+2} \delta_{k}\right) \times\left(1-\frac{\Omega^{2}}{\delta_{1} \delta_{2}}-\sum_{l=1}^{D} \frac{c_{l}^{2}}{\delta_{2} \delta_{l+2}}\right) .
$$

The eigenvalue equation, $\operatorname{det}(H-\lambda \mathbb{I})=0$, is obtained through the replacement $\delta_{k} \rightarrow \delta_{k}-\lambda$ :

$$
P(\lambda) \equiv\left[\prod_{k=1}^{D+2}\left(\delta_{k}-\lambda\right)\right] \times\left[1-\frac{\Omega^{2}}{\left(\delta_{1}-\lambda\right)\left(\delta_{2}-\lambda\right)}-\sum_{l=1}^{D} \frac{c_{l}^{2}}{\left(\delta_{2}-\lambda\right)\left(\delta_{l+2}-\lambda\right)}\right]=0 .
$$

Considering $\lambda=\delta_{1}+\sigma$, one gets:

$$
P\left(\delta_{1}+\sigma\right)=-\sigma\left[\prod_{k=3}^{D+2}\left(\delta_{k}-\delta_{1}-\sigma\right)\right] \times\left[\delta_{2}-\delta_{1}-\sigma+\frac{\Omega^{2}}{\sigma}-\sum_{l=1}^{D} \frac{c_{l}^{2}}{\delta_{l+2}-\delta_{1}-\sigma}\right]=0 .
$$

The eigenvalue close to $\delta_{1}$ - Let us first consider the case where $\delta_{k+2}>\delta_{1} \quad \forall k \geq 1$.

In the limit $\sigma \rightarrow 0$ one obtains:

$$
P\left(\delta_{1}\right)=-\Omega^{2}\left[\prod_{k=3}^{D+2}\left(\delta_{k}-\delta_{1}\right)\right]
$$

from which one immediately finds $P\left(\delta_{1}\right)<0$.

On the other hand, when $c$ is large enough one can prove that $P\left(\delta_{1}+\xi\right)>0$ for $\xi=2 M \Omega^{2} / c^{2}$. Indeed, assume $c^{2}>2 M \Omega^{2} / m$ so that $\delta_{l+2}-\delta_{1}-\xi>0 \quad \forall k \geq$ 1. Therefore, since $M>\delta_{l+2}-\delta_{1}-\xi \quad \forall l$ and hence $\sum_{l} c_{l}^{2} /\left(\delta_{l+2}-\delta_{1}-\xi\right)>\sum_{l} c_{l}^{2} / M=c^{2} / M$, one obtains:

$$
\delta_{2}-\delta_{1}-\xi+\frac{\Omega^{2}}{\xi}-\sum_{l=1}^{D} \frac{c_{l}^{2}}{\delta_{l+2}-\delta_{1}-\xi}<\delta_{2}-\delta_{1}-\frac{c^{2}}{2 M} .
$$

Now, if $\delta_{2}-\delta_{1}<0$ then we have a negative quantity for any $c$, and therefore $P\left(\delta_{1}+\xi\right)>0$. If instead $\delta_{2}-\delta_{1} \geq 0$, we need to take $c>\left(2\left(\delta_{2}-\delta_{1}\right) M\right)^{1 / 2}$. Of course we always have to satisfy the previously considered condition $c^{2}>2 M \Omega^{2} / m$ to guarantee $\delta_{k+2}-\delta_{1}-\xi>0$.

Summarizing, for $c>\max \left\{\Omega \sqrt{2 M / m},\left[2\left(\delta_{2}-\right.\right.\right.$ $\left.\left.\left.\delta_{1}\right) M\right]^{1 / 2}\right\}$ and $\xi=2 M \Omega^{2} / c^{2}$ we are sure that $P\left(\delta_{1}+\xi\right)>$ 0 , and we can then assert that for some $\eta: 0<\eta<\xi=$ $2 M \Omega^{2} / c^{2}$ there is an eigenvalue $\lambda_{1}=\delta_{1}+\eta$.

Eigenstate and overlap - If $\Omega \neq 0$ and $\delta_{k+2}-\delta_{1}-\eta \neq$ $0 \forall k$, then one can easily find that the corresponding eigenstate is:

$$
\begin{aligned}
\left|\delta_{1}+\eta\right\rangle & =\aleph_{\eta}\left[|A\rangle+\frac{\eta}{\Omega}|B\rangle\right. \\
& \left.-\sum_{l=1}^{D} \frac{\Omega^{-1} c_{l} \eta}{\left(\delta_{l+2}-\delta_{1}-\eta\right)}\left|C_{l}\right\rangle\right],
\end{aligned}
$$

which approaches $|A\rangle$ in the limit of very large $c$. Indeed, consider first of all that in such limit we have $\eta \rightarrow 0$, since $0<\eta<\xi=2 M \Omega^{2} / c^{2} \rightarrow 0$, so that the condition $\delta_{l+2}-\delta_{1}-\eta \neq 0$ is easily satisfied. Secondly, one finds 
$\left|c_{l} \eta\right| \leq\left|2 M \Omega^{2} c_{l} / 2 c^{2}\right| \leq 2 M \Omega^{2} / c \rightarrow 0$, and on this basis it is immediate to prove that $\left|\left\langle\delta_{1}+\eta \mid A\right\rangle\right| \rightarrow 1$.

Now, one cannot be sure that $\delta_{l+2}-\delta_{1}-\xi>m$, even for small $\xi$, but assuming $c^{2}>4 M \Omega^{2} / m$ one has $\left(\delta_{l+2}-\right.$ $\left.\delta_{1}-\xi\right)^{-1}<2 / m$ and a fortiori $\left(\delta_{l+2}-\delta_{1}-\eta\right)^{-1}<2 / m$. Then, reminding that $\eta<2 M \Omega^{2} / c$, one finds:

$$
\begin{aligned}
\left\langle\delta_{1}+\eta \mid A\right\rangle & =\left(1+\frac{\eta^{2}}{\Omega^{2}}+\sum_{l=1}^{D} \frac{\Omega^{-2} \eta^{2} c_{l}^{2}}{\left(\delta_{l+2}-\delta_{1}-\eta\right)^{2}}\right)^{-\frac{1}{2}} \\
& \geq\left(1+\frac{4 M^{2} \Omega^{2}}{c^{4}}+\sum_{l=1}^{D} \frac{16 M^{2} \Omega^{2} c_{l}^{2}}{m^{2} c^{4}}\right)^{-\frac{1}{2}} \\
& =\left(1+\frac{4 M^{2} \Omega^{2}}{c^{4}}+\frac{16 M^{2} \Omega^{2}}{m^{2} c^{2}}\right)^{-\frac{1}{2}} \\
& =\frac{m c^{2}}{\left(m^{2} c^{4}+16 M^{2} \Omega^{2} c^{2}+4 m^{2} M^{2} \Omega^{2}\right)^{1 / 2}}
\end{aligned}
$$

which clearly approaches unity in the limit $c \rightarrow \infty$. All this reasoning is invalidated if $m=0$.

The condition

$$
\left|\left\langle\delta_{1}+\eta \mid A\right\rangle\right| \geq \sqrt{\chi}
$$

is satisfied when:

$$
c \geq \frac{4 \Omega M \sqrt{\chi}}{m} \sqrt{\frac{1+\sqrt{1+(1-\chi) m^{4} /\left(16 \chi M^{2} \Omega^{2}\right)}}{2(1-\chi)}} .
$$

Assuming $m / \Omega$ not diverging and $\chi \rightarrow 1$, we can neglect the term multiplying $1-\chi$, then requesting just the following:

$$
c \gtrsim \frac{4 M \Omega}{m} \sqrt{\frac{\chi}{1-\chi}} .
$$

Of course, we also need to have $c>$ $\max \left\{4 M \Omega^{2} / m,\left[2\left(\delta_{2}-\delta_{1}\right) M\right]^{1 / 2}\right\}$ - which includes the previously considered $c^{2}>2 M \Omega^{2} / m-$, but for $\chi$ close enough to unity such condition is surely included in the condition (B13).

Generalization and limitations - All the previous discussion keeps its validity if $\delta_{k+2}<\delta_{1} \quad \forall k \geq 1$, which means that $\prod_{l=3}^{D+2}\left(\delta_{l}-\delta_{1}\right)$ appearing in $P\left(\delta_{1}\right)$ - see Eq. (B7) - has the sign $(-1)^{D}$. Now, define $\xi^{\prime}=$ $-2 M \Omega^{2} / c^{2}$, and assume $c^{2}>2 M \Omega^{2} / m$ in order to have $\delta_{k+2}-\delta_{1}-\xi^{\prime}<0 \quad \forall k$, so that $\prod_{l=3}^{D+2}\left(\delta_{l}-\delta_{1}-\xi\right)$ maintains its previous sign. Since:

$$
\delta_{2}-\delta_{1}-\xi^{\prime}+\frac{\Omega^{2}}{\xi^{\prime}}-\sum_{l=1}^{D} \frac{c_{l}^{2}}{\delta_{l+2}-\delta_{1}-\xi^{\prime}}>\delta_{2}-\delta_{1}+\frac{c^{2}}{2 M},
$$

in order to obtain a change of sign for the quantity $P\left(\delta_{1}+\right.$ $\xi)$ it is sufficient to have:

$$
\delta_{2}-\delta_{1}+\frac{c^{2}}{2 M}>0
$$

Now, either $\delta_{2}-\delta_{1} \geq 0$, so that the quantity on the right-hand side above is positive whatever the number $c$, or $\delta_{2}-\delta_{1}<0$, and that quantity can be made positive provided $c>\left(2\left|\delta_{2}-\delta_{1}\right| M\right)^{1 / 2}$. This immediately implies that $P\left(\delta_{1}\right)$ and $P\left(\delta_{1}+\xi^{\prime}\right)$ have opposite signs, provided $c$ large enough. Therefore, this time we have an eigenvalue $\lambda_{1}=\delta_{1}+\eta^{\prime}$ with $-2 M \Omega^{2} / c^{2}<\eta^{\prime}<0$. The corresponding eigenstate $\left|\delta_{1}+\eta^{\prime}\right\rangle$ is close to $|A\rangle$, since the inequality we derived for $\left|\delta_{1}+\eta\right\rangle$ in (B10) is valid also for $\left|\delta_{1}+\eta^{\prime}\right\rangle$.

Here we are not considering those cases in which $\delta_{l+2}>$ $\delta_{1}$ holds for some $l$ 's and does not hold for others.

Finally, we remark that if the $c_{k}$ 's are not real - corresponding to the interaction $\sum_{k}\left(g_{k} \hat{a}_{k}|2\rangle\left\langle 3\left|+g_{k}^{*} \hat{a}_{k}^{\dagger}\right| 3\right\rangle\langle 2|\right)$, which generalizes that in Eq. (9) - the previous results keep holding, provided the replacement $c_{k}^{2} \rightarrow\left|c_{k}\right|^{2}$ everywhere.

\section{Appendix C}

The geometric factor $G_{D}$ in Eq. (21) comes from the right hand-side integral of Eq. (20). In particular, exploiting to hyperspherical coordinates, such integral requires integration over the $D-1$ spherical angles. In general there are $D-2$ angles which can run over $[0, \pi]$ and one that can run over $[0,2 \pi]$. In our particular case, the spanned intervals are smaller, since the variables $y_{j}=g_{j}^{2} n_{j}$ can assume only positive values, and hence all the angles span $[0, \pi / 2]$.

The spherical coordinates are defined by:

$$
\begin{aligned}
y_{1} & =r \cos \phi_{1}, \\
y_{2} & =r \sin \phi_{1} \cos \phi_{2}, \\
y_{3} & =r \sin \phi_{1} \sin \phi_{2} \cos \phi_{3}, \\
\vdots & \\
y_{D-1} & =r \sin \phi_{1} \sin \phi_{2} \ldots \sin \phi_{D-2} \cos \phi_{D-1} \\
y_{D} & =r \sin \phi_{1} \sin \phi_{2} \ldots \sin \phi_{D-2} \sin \phi_{D-1},
\end{aligned}
$$

which correspond to the volume element:

$$
\prod_{k} \mathrm{~d} y_{k}=r^{D-1}\left(\prod_{k=1}^{D-2} \sin ^{D-k-1} \phi_{k}\right) \times \mathrm{d} r \prod_{k=1}^{D-1} \mathrm{~d} \phi_{k}
$$

that can be straightforwardly derived from the Jacobian of the coordinate transformation $\left|\partial y_{k} / \partial x_{j}\right|$, with $x_{j}=\phi_{j}$ for $j \leq D-1$ and $x_{D}=r$. 
Therefore one obtains:

$$
\begin{aligned}
& \int_{y_{n} \geq 0} \cdots \int_{\sum_{n} y_{n}^{2}<c_{\varepsilon}} \prod_{j=1}^{D} y_{j} \mathrm{~d} y_{j} \\
& =\int_{0}^{c_{\varepsilon}} r^{2 D-1} \mathrm{~d} r \int \ldots \int \prod_{k=1}^{D-1} f_{k}\left(\phi_{k}\right) \mathrm{d} \phi_{k} \\
& =\frac{c_{\varepsilon}^{2 D}}{2 D} \int_{0}^{\frac{\pi}{2}} \cdots \int_{0}^{\frac{\pi}{2}} \prod_{k=1}^{D-1} \sin ^{2(D-k)-1} \phi_{k} \cos \phi_{k} \mathrm{~d} \phi_{k} \\
& =\frac{c_{\varepsilon}^{2 D}}{2 D} \times \frac{1}{2^{D-1}(D-1) !}=\frac{c_{\varepsilon}^{2 D}}{2^{D} D !} .
\end{aligned}
$$

On this basis we find that the geometric factor raising from the integration over the angles is:

$$
G_{D}=\frac{1}{2^{D} D !}
$$

Exploiting the Stirling approximation [26], which is valid for large $D$, one eventually gets:

$$
G_{D} \approx(2 \pi D)^{-1 / 2} 2^{-D} D^{-D} \mathrm{e}^{D}
$$

[1] B. Misra and E. C. G. Sudarshan, J. Math. Phys. 18, 7456 (1997).

[2] W. M. Itano, D. J. Heinzen, J. J. Bollinger and D. J. Wineland, Phys. Rev. A 412295 (1990).

[3] S. R. Wilkinson, C. F. Bharucha, M. C. Fischer, K. W. Madison, P. R. Morrow, Qian Niu, Bala Sundaram, and M. G. Raizen, Nature 387, 575 (1997).

[4] L. S. Schulman, Phys. Rev. A 57, 1509 (1998).

[5] A. D. Panov, Phys. Lett. A 260, 441 (1999).

[6] J. Audretsch, M. B. Mensky, A. D. Panov, Phys.Lett. A 261, 44 (1999).

[7] B. Militello, M. Scala, A. Messina and N. V. Vitanov, Phys. Scr. T 143, 014019 (2011).

[8] M. Scala, B. Militello, A. Messina, and N. V. Vitanov, Phys. Rev. A 81, 053847 (2010).

[9] P. Facchi and S. Pascazio, Progress in Optics 41 edited by E. Wolf, Elsevier, Amsterdam, 2001.

[10] B. Militello, A. Messina and A. Napoli, J. Phys. A 286, 369 (2001).

[11] B. Militello, A. Messina and A. Napoli, Fortscr. Phys. 49, 1041 (2001).

[12] P. Facchi and S. Pascazio, Phys. Rev. Lett. 89, 080401 (2002).

[13] P. Facchi, D. A. Lidar and S. Pascazio, Phys. Rev. A 69, 032314 (2004).

[14] P. Facchi and S. Pascazio, J. Phys. A: Math. Theor. 41,
493001 (2008).

[15] P. Facchi, G. Marmo and S. Pascazio, J. Phys.: Conf. Ser. 196, 012017 (2009).

[16] A. Peres, Am. J. Phys. 48, 931 (1980).

[17] D. Home and M. A. B. Whitaker, Ann. Phys. 258, 237

[18] Keisuke Fujii and Katsuji Yamamoto, Phys. Rev. A 82, 042109 (2010).

[19] Gonzalo Alvarez, D. D. Bhaktavatsala Rao, L. Frydman, and G. Kurizki, Phys. Rev. Lett. 105, 160401 (2010).

[20] R. Schützhold and G. Gnanapragasam, Phys. Rev. A 82 , 022120 (2010).

[21] J. Ruseckas, Phys. Rev. A 66, 012105 (2002).

[22] S. Maniscalco, J. Piilo and K.-A. Suominen, Phys. Rev. Lett. 97, 130402 (2006).

[23] D. D. Bhaktavatsala Rao and Gershon Kurizki, Phys. Rev. A 83, 032105 (2011).

[24] C. W. Gardiner and P. Zoller, Quantum Noise (SpringerVerlag, Berlin, 2000).

[25] H.-P. Breuer and F. Petruccione, The Theory of Open Quantum Systems (Oxford University Press, Oxford, 2002).

[26] M. Abramowitz and I. A. Stegun, Handbook of Mathematical Functions (Dover Publications, 1965). (1997). 